\title{
FORMULATION OF THE HEAT CONDUCTION EQUATION FOR HETEROGENEOUS MEDIA WITH MULTIPLE SPATIAL SCALES USING REITERATED HOMOGENIZATION
}

\author{
E. Iglesias-Rodríguez ${ }^{\mathrm{a}}$, \\ M. E. Cruz ${ }^{b}$, \\ J. Bravo-Castillero ${ }^{c}$, \\ R. Guinovart-Díaz ${ }^{c}$, \\ R. Rodríguez-Ramos ${ }^{c}$, \\ and L. D. Pérez-Fernández ${ }^{d}$

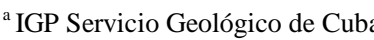 \\ Vía Blanca 1002, CP 11000, La Habana, Cuba \\ ${ }^{\text {b} U n i v e r s i d a d e ~ F e d e r a l ~ d e R i o ~ d e ~ J a n e i r o ~}$ \\ Departamento de Engenharia Mecânica \\ Politécnica/COPPE, CT, CP 68503 \\ Cidade Universitária, Rio de Janeiro-RJ, \\ 21941-972, Brasil \\ manuel@mecanica.coppe.ufrj.br \\ ${ }^{c}$ Universidad de La Habana \\ Departamento de Matemática \\ Facultad de Matemática y Computación \\ San Lázaro y L, La Habana, CP 10400, Cuba \\ ${ }^{\mathrm{d}}$ Universidade Federal de Pelotas \\ Departamento de Matemática e Estatística \\ Instituto de Física e Matemática \\ CP 354, Pelotas-RS, 96010-971, Brasil \\ Received: April 02, 2016 \\ Revised: May 16, 2016 \\ Accepted: June 13, 2016
}

\section{ABSTRACT}

Heterogeneous media with multiple spatial scales are finding increased importance in engineering. An example might be a large scale, otherwise homogeneous medium filled with dispersed small-scale particles that form aggregate structures at an intermediate scale. The objective in this paper is to formulate the strong-form Fourier heat conduction equation for such media using the method of reiterated homogenization. The phases are assumed to have a perfect thermal contact at the interface. The ratio of two successive length scales of the medium is a constant small parameter $\varepsilon$. The method is an up-scaling procedure that writes the temperature field as an asymptotic multiple-scale expansion in powers of the small parameter $\varepsilon$. The technique leads to two pairs of local and homogenized equations, linked by effective coefficients. In this manner the medium behavior at the smallest scales is seen to affect the macroscale behavior, which is the main interest in engineering. To facilitate the physical understanding of the formulation, an analytical solution is obtained for the heat conduction equation in a functionally graded material (FGM). The approach presented here may serve as a basis for future efforts to numerically compute effective properties of heterogeneous media with multiple spatial scales.

Keywords: heat conduction, heterogeneous media, reiterated

homogenization, formal asymptotic solution, functionally graded material

\section{NOMENCLATURE}

$f \quad$ density of the heat sources, $\mathrm{W} / \mathrm{m}^{3}$

K conductivity tensor, $\mathrm{W} /(\mathrm{m} \cdot \mathrm{K})$

$\hat{\mathbf{K}}$ effective conductivity tensor, $\mathrm{W} /(\mathrm{m} \cdot \mathrm{K})$

$T$ temperature, ${ }^{\circ} \mathrm{C}$

$\mathbf{x} \quad$ spatial variable at the macroscale, $\mathrm{m}$

y spatial variable at the first microscale, $\mathrm{m}$

$Y \quad$ Periodic cell at the first microscale

$\mathbf{z} \quad$ spatial variable at the second microscale, $\mathrm{m}$

$Z \quad$ Periodic cell at the second microsscale

\section{Greek symbols}

$\varepsilon \quad$ dimensionless small positive parameter

X solution of local problems on $Y$

$$
\begin{array}{ll}
\mathbf{X}_{y} & \text { solution of local problems on } Z \text { for } y \in Y \\
\Omega & \text { an open connected bounded subset of } \square^{3} \\
\partial \Omega & \text { boundary of } \Omega
\end{array}
$$

\section{INTRODUCTION}

In some recent engineering applications it has become important to know the macroscopic behavior of heterogeneous materials in terms of several different microscopic levels. Reiterated homogenization is a mathematical technique for deriving the effective coefficients for such type of complex media. From the mathematical point of view, the homogenization process allows to transform problems involving systems of partial differential equations with rapidly oscillating coefficients in others, wherein the coefficients are not 
rapidly oscillating, and are the so-called effective coefficients. To demonstrate proximity between the solutions of the original and the homogenized problems is the key for the mathematical justification. Classical studies of the linear elliptic partial differential equation can be found, for instance, in Panasenko (2008) for two-scale media, and in Bensoussan et al. (1978) and Allaire and Briane (1996) for media with two or more spatial scales.

As an application-oriented study, the aim of this work is to; first, describe didactically the formal procedure of the reiterated homogenization method for the Fourier heat conduction equation with differentiable coefficients. Second, the formulation is employed to obtain the effective properties of a conductive functionally graded material -- FGM (Mahamood et al., 2012; Paulino et al., 2009; Yin et al., 2005) with periodic, rapidly oscillating, isotropic, and infinitely differentiable coefficients. The approach presented here may serve as a basis for future efforts to numerically compute effective properties of heterogeneous media with multiple spatial scales, and may be useful for the development of behavioral models to be embodied in a broader multi-material design method (Wargnier et al., 2014).

Let $\Omega$ be a three-dimensional open connected bounded subset with a sufficiently smooth boundary $\partial \Omega$. The domain $\Omega$ extends over the spatial coordinate $\mathbf{x}$. Two structural levels are considered, such that $\varepsilon$ denotes the small positive parameter that is the ratio of two successive length scales of the medium. Thus, the fast variables $\mathbf{y}$ and $\mathbf{z}$ are defined, $\mathbf{y}=\mathbf{x} / \varepsilon, \mathbf{z}=\mathbf{y} / \varepsilon=\mathbf{x} / \varepsilon^{2}$. The components of the conductivity tensor $k_{i j}^{\varepsilon}(\mathbf{x})=k_{i j}(\mathbf{x}, \varepsilon)=k_{i j}(\mathbf{y}, \mathbf{z})$ are $Y \times Z$-periodic differentiable functions.

The formalism of the reiterated homogenization method is here applied to the following family of boundary value problems depending on $\varepsilon$,

$$
\begin{gathered}
\frac{\partial}{\partial x_{i}}\left(k_{i j}^{\varepsilon} \frac{\partial T^{\varepsilon}}{\partial x_{j}}\right)=f, \text { in } \Omega \\
T^{\varepsilon}=0 \text {, on } \partial \Omega
\end{gathered}
$$

where $T^{\varepsilon}(\mathbf{x})$ is a scalar field, $\mathbf{K}=\left(k_{i j}^{\varepsilon}\right)$ is a symmetric and positive definite matrix-valued function, and $f \in C^{\infty}(\Omega)$. Physically, problem (1)(2) may govern the temperature field $T^{\varepsilon}$ set up by heat conduction in a medium with thermal conductivity $\mathbf{K}$.

By applying the reiterated homogenization method, the family of problems (1)-(2) yields

$$
\hat{k}_{i j} \frac{\partial^{2} T}{\partial x_{i} \partial x_{j}}=f \text {, in } \Omega
$$

$$
T^{\mathcal{E}}=0 \text {, on } \partial \Omega
$$

where $\hat{\mathbf{K}}=\left(\hat{k}_{i j}\right)$ is a symmetric, positive definite matrix with constant entries. The new problem (3)-(4) is called the homogenized problem. The entries of $\hat{\mathbf{K}}$ are the effective coefficients which determine the macroscopic behavior of the heterogeneous medium.

\section{HOMOGENIZATION: LOCAL AND HOMOGENIZED PROBLEMS, AND EFFECTIVE COEFFICIENTS}

In this section, following Bensoussan et al. (1978) and Bakhvalov and Panasenko (1989), the reiterated homogenization method is applied to the problem (1)-(2). The following operators and notations are used in this section:

$$
\begin{aligned}
L_{p q} & =\frac{\partial}{\partial p_{i}}\left(k_{i j}^{\varepsilon} \frac{\partial}{\partial q_{j}}\right), \\
L^{1} & =L_{z z}, \quad L^{2}=L_{y z}+L_{z y}, \quad L^{3}=L_{x z}+L_{y y}+L_{z x}, \\
L^{4} & =L_{x y}+L_{y x}, \quad L^{5}=L_{x x}
\end{aligned}
$$

The formal asymptotic solution of (1)-(2) is sought in the form

$$
\begin{aligned}
T^{\varepsilon}(\mathbf{x}) & =T_{0}(\mathbf{x}, \mathbf{y}, \mathbf{z})+\varepsilon T_{1}(\mathbf{x}, \mathbf{y}, \mathbf{z})+\varepsilon^{2} T_{2}(\mathbf{x}, \mathbf{y}, \mathbf{z}) \\
& +\varepsilon^{3} T_{3}(\mathbf{x}, \mathbf{y}, \mathbf{z})+\varepsilon^{4} T_{4}(\mathbf{x}, \mathbf{y}, \mathbf{z})+\ldots,
\end{aligned}
$$

where $T_{i}$ are differentiable functions, and $Y$-periodic with respect to $\mathbf{y}$ and $Z$-periodic with respect to $\mathbf{z}$. The operator $\partial / \partial x_{i}$ for a function $v=\hat{v}(\mathbf{x})=\tilde{v}(\mathbf{x}, \mathbf{y}, \mathbf{z})$ is thus determined using the chain rule,

$$
\frac{\partial v}{\partial x_{i}}=\frac{\partial \hat{v}}{\partial x_{i}}=\frac{\partial \tilde{v}}{\partial x_{i}}+\frac{\partial \tilde{v}}{\partial y_{i}} \frac{\partial y_{i}}{\partial x_{i}}+\frac{\partial \tilde{v}}{\partial z_{i}} \frac{\partial z_{i}}{\partial x_{i}},
$$

such that

$$
\frac{\partial}{\partial x_{i}}=\frac{\partial}{\partial x_{i}}+\frac{1}{\varepsilon} \frac{\partial}{\partial y_{i}}+\frac{1}{\varepsilon^{2}} \frac{\partial}{\partial z_{i}} .
$$

Applying Eq. (7) to the left-hand side of Eq. (1) yields

$$
\begin{array}{r}
\frac{\partial}{\partial x_{i}}\left(k_{i j}^{\varepsilon} \frac{\partial T^{\varepsilon}}{\partial x_{j}}\right)=\left(\frac{\partial}{\partial x_{i}}+\frac{1}{\varepsilon} \frac{\partial}{\partial y_{i}}+\frac{1}{\varepsilon^{2}} \frac{\partial}{\partial z_{i}}\right) \\
\times\left(k_{i j}^{\varepsilon}\left(\frac{\partial T^{\varepsilon}}{\partial x_{j}}+\frac{1}{\varepsilon} \frac{\partial T^{\varepsilon}}{\partial y_{j}}+\frac{1}{\varepsilon^{2}} \frac{\partial T^{\varepsilon}}{\partial z_{j}}\right)\right),
\end{array}
$$

or, equivalently, 


$$
\begin{aligned}
\frac{\partial}{\partial x_{i}}\left(k_{i j}^{\varepsilon} \frac{\partial T^{\varepsilon}}{\partial x_{j}}\right) & =\varepsilon^{-4} L^{1} T^{\varepsilon}+\varepsilon^{-3} L^{2} T^{\varepsilon} \\
& +\varepsilon^{-2} L^{3} T^{\varepsilon}+\varepsilon^{-1} L^{4} T^{\varepsilon}+\varepsilon^{0} L^{5} T^{\varepsilon} .
\end{aligned}
$$

Using Eq. (7), Eq. (1) becomes

$$
\left(\varepsilon^{-4} L^{1}+\varepsilon^{-3} L^{2}+\varepsilon^{-2} L^{3}+\varepsilon^{-1} L^{4}+\varepsilon^{0} L^{5}\right) T^{\varepsilon}=f .
$$

Substituting the asymptotic expansion of $T^{\mathcal{E}}$ up to $\varepsilon^{4}$, Eq. (5), into Eq. (10), one obtains

$$
\begin{aligned}
\left(\varepsilon^{-4} L^{1}\right. & \left.+\varepsilon^{-3} L^{2}+\varepsilon^{-2} L^{3}+\varepsilon^{-1} L^{4}+\varepsilon^{0} L^{5}\right) \\
& \times\left(T_{0}+\varepsilon T_{1}+\varepsilon^{2} T_{2}+\varepsilon^{3} T_{3}+\varepsilon^{4} T_{4}\right)=f .
\end{aligned}
$$

Equating to zero the terms corresponding to equal powers of $\varepsilon$, up to $\varepsilon^{0}$, the following recurrent chain of equalities is obtained:

$$
\begin{gathered}
\text { from } \varepsilon^{-4}, L^{1} T_{0}=0, \\
\text { from } \varepsilon^{-3}, L^{1} T_{1}=-L^{2} T_{0}, \\
\text { from } \varepsilon^{-2}, L^{1} T_{2}=-L^{2} T_{1}-L^{3} T_{0}, \\
\text { from } \varepsilon^{-1}, L^{1} T_{3}=-L^{2} T_{2}-L^{3} T_{1}-L^{4} T_{0}, \\
\text { from } \varepsilon^{0}, L^{1} T_{4}=-L^{2} T_{3}-L^{3} T_{2}-L^{4} T_{1}-L^{5} T_{0}+f .
\end{gathered}
$$

Note that all the equations (12)-(16) have a similar form,

$$
L^{1} U=F+\frac{\partial G_{i}}{\partial z_{i}}
$$

where $L^{1}$ involves $\mathbf{z}$ only, and $U$ and $G_{i}$ are $Z$ periodic. For Eq. (15), Bakhvalov and Panasenko (1989) proved that

$$
\langle F\rangle_{Z}=0
$$

is a necessary and sufficient condition for the existence of a periodic solution, and the latter is unique perhaps up to an additive constant. The angular brackets denote the average of the function $F$ over the periodic cell $Z\left(|Z|^{-1} \int_{Z} F d \mathbf{z}\right)$.

By applying condition (18) to Eq. (12), one concludes that $T_{0}$ is a function of $\mathbf{x}$ and $\mathbf{y}$ only, or

$$
T_{0}=\tilde{T}_{0}(\mathbf{x}, \mathbf{y}) .
$$

Inserting Eq. (19) into Eq. (13), one obtains

$$
L^{1} T_{1}=-\frac{\partial k_{i j}}{\partial z_{i}} \frac{\partial \tilde{T}_{0}}{\partial y_{j}},
$$

such that a solution to Eq. (20) can be represented as

$$
T_{1}(\mathbf{x}, \mathbf{y}, \mathbf{z})=-\chi_{y}^{\ell}(\mathbf{z}) \frac{\partial \tilde{T}_{0}}{\partial y_{\ell}}(\mathbf{x}, \mathbf{y})+\tilde{T}_{1}(\mathbf{x}, \mathbf{y})
$$

where $\chi_{y}^{\ell}(\mathbf{z})$ is the solution of the local problem, or cell problem, described next.

First Local Problem: For each $\mathbf{y} \in Y$, find the parametric family of $Z$-periodic functions $\left\{\chi_{y}^{j}(\mathbf{z})\right\}_{y \in Y}$, such that

$$
\begin{gathered}
-\frac{\partial}{\partial z_{i}}\left(k_{i j}-k_{i l} \frac{\partial \chi_{y}^{j}(\mathbf{z})}{\partial z_{l}}\right)=0, \quad \text { in } \mathrm{Z}, \\
\left\langle\chi_{y}^{\ell}(\mathbf{z})\right\rangle_{z}=0 .
\end{gathered}
$$

With Eq. (19), Eq. (14) simplifies to

$$
L^{1} T_{2}=-L_{z y} T_{1}-L_{y z} T_{1}-L_{z x} \tilde{T}_{0}-L_{y y} \tilde{T}_{0},
$$

which, given Eq. (21) can be rewritten as

$$
\begin{aligned}
L^{1} T_{2}= & -L_{z y} T_{1}-L_{z x} \tilde{T}_{0} \\
& -L_{y y} \tilde{T}_{0}+\frac{\partial}{\partial y_{i}}\left(k_{i j} \frac{\partial \chi_{y}^{\ell}}{\partial z_{j}} \frac{\partial \tilde{T}_{0}}{\partial y_{\ell}}\right)
\end{aligned}
$$

From Eq. (18), the necessary and sufficient condition to be held for the solvability of Eq. (25) in the class of $Z$-periodic functions is

$$
\left\langle-L_{y y} \tilde{T}_{0}+\frac{\partial}{\partial y_{i}}\left(k_{i j} \frac{\partial \chi_{y}^{\ell}}{\partial z_{j}} \frac{\partial \tilde{T}_{0}}{\partial y_{\ell}}\right)\right\rangle_{z}=0
$$

or, removing the $y$-derivatives and using Eq. (19),

$$
-\frac{\partial}{\partial y_{i}}\left(\left\langle k_{i l}-k_{i j} \frac{\partial \chi_{y}^{\ell}}{\partial z_{j}}\right\rangle_{z} \frac{\partial \tilde{T}_{0}}{\partial y_{\ell}}\right)=0 .
$$

Next, by introducing the operator

$$
\Lambda \equiv-\frac{\partial}{\partial y_{i}}\left(k_{i j}^{1}(\mathbf{y}) \frac{\partial}{\partial y_{j}}\right)
$$


with

$$
k_{i l}^{1}(\mathbf{y}) \equiv\left\langle k_{i l}-k_{i j} \frac{\partial \chi_{y}^{\ell}}{\partial z_{j}}\right\rangle_{Z},
$$

Eq. (27) takes the more compact form

$$
\Lambda \tilde{T}_{0}=0 .
$$

Eq. (30) constitutes the intermediate homogenized problem, whose effective thermal conductivity tensor is $k_{i j}^{1}(\mathbf{y})$, which preserves the symmetry and positive definiteness properties of $k_{i j}$.

Using a similar argument as that for the deduction of Eq. (19) from Eq. (12), one concludes from Eq. (30) that

$$
\tilde{T}_{0}=T(\mathbf{x}) .
$$

Substituting Eq. (31) into Eq. (21) for $T_{1}$ yields

$$
T_{1}=\tilde{T}_{1}(\mathbf{x}, \mathbf{y})
$$

Now substituting Eq. (31) and Eq. (32) into Eq. (25) yields

$$
L^{1} T_{2}=-\frac{\partial k_{i j}(\mathbf{y}, \mathbf{z})}{\partial z_{i}}\left(\frac{\partial T}{\partial x_{j}}+\frac{\partial \tilde{T}_{1}}{\partial y_{j}}\right)
$$

In analogy to Eqs. (20)-(21), a solution to Eq. (33) can be represented as

$$
\begin{aligned}
T_{2}(\mathbf{x}, \mathbf{y}, \mathbf{z})= & -\chi_{y}^{l}(\mathbf{z})\left(\frac{\partial T(\mathbf{x})}{\partial x_{l}}+\frac{\partial \tilde{T}_{1}(\mathbf{x}, \mathbf{y})}{\partial y_{l}}\right) . \\
& +\tilde{T}_{2}(\mathbf{x}, \mathbf{y})
\end{aligned}
$$

Considering Eq. (31) and Eq. (32), Eq. (15) transforms to

$$
L^{1} T_{3}=-L_{y z} T_{2}-L_{z y} T_{2}-L_{y y} \tilde{T}_{1}-L_{z x} \tilde{T}_{1}-L_{y x} T .
$$

Substituting into Eq. (35) the expression (34) for $T_{2}$ yields

$$
\begin{aligned}
L^{1} T_{3} & =\frac{\partial}{\partial y_{i}}\left(k_{i j} \frac{\partial \chi_{y}^{l}}{\partial z_{j}}\left(\frac{\partial T}{\partial x_{l}}+\frac{\partial \tilde{T}_{1}}{\partial y_{l}}\right)\right) \\
& -\frac{\partial}{\partial y_{i}}\left(k_{i j} \frac{\partial \tilde{T}_{1}}{\partial y_{j}}\right)-\frac{\partial}{\partial y_{i}}\left(k_{i j} \frac{\partial \tilde{T}_{1}}{\partial x_{j}}\right) \\
& -\frac{\partial}{\partial z_{i}}\left(-k_{i j} \chi_{y}^{l} \frac{\partial^{2} \tilde{T}_{1}}{\partial y_{j} \partial y_{l}}+k_{i j} \frac{\partial \tilde{T}_{2}}{\partial y_{j}}+k_{i j} \frac{\partial \tilde{T}_{1}}{\partial x_{j}}\right)
\end{aligned}
$$

(18) to Eq. (3), one obtains

$$
\begin{aligned}
& \left\{-\frac{\partial}{\partial y_{i}} \frac{1}{|Z|}\left(\int_{Z} k_{i j} d \mathbf{z}-\int_{Z} k_{i l} \frac{\partial \chi_{y}^{j}}{\partial z_{l}} d \mathbf{z}\right) \frac{\partial}{\partial y_{j}}\right\} \tilde{T}_{1} \\
& =\frac{\partial T}{\partial x_{p}}\left\{\frac{\partial}{\partial y_{i}} \frac{1}{|Z|}\left(\int_{Z} k_{i j} d \mathbf{z}-\int_{Z} k_{i l} \frac{\partial \chi_{y}^{j}}{\partial z_{l}} d \mathbf{z}\right) \frac{\partial}{\partial y_{j}}\right\} y_{p}
\end{aligned}
$$
becomes

Using the definition of the operator $\Lambda$, Eq. (37)

$$
\Lambda \tilde{T}_{1}=-\left(\Lambda y_{j}\right) \frac{\partial T}{\partial x_{j}}
$$

It is now possible to separate variables $\mathbf{x}$ and $\mathbf{y}$, and write $\tilde{T}_{1}$ as

$$
\tilde{T}_{1}=-\chi^{j}(\mathbf{y}) \frac{\partial T(\mathbf{x})}{\partial x_{j}}+\tilde{\tilde{T}}_{1}(\mathbf{x})
$$

The expression (34) for $T_{2}$ thus becomes

$$
\begin{aligned}
T_{2} & =-\chi_{y}^{l}(\mathbf{z})\left(\frac{\partial T(\mathbf{x})}{\partial x_{l}}+\frac{\partial \chi^{j}(\mathbf{y})}{\partial y_{l}} \frac{\partial T(\mathbf{x})}{\partial x_{j}}\right) \\
& +\tilde{T}_{2}(\mathbf{x}, \mathbf{y})
\end{aligned}
$$

Substituting Eq. (39) into Eq. (38), one obtains the local problem, or cell problem, described next.

Second Local Problem: Find $\chi^{j}, Y$-periodic, such that

$$
\Lambda\left(\chi^{j}-y_{j}\right) \equiv-\frac{\partial}{\partial y_{i}}\left(k_{i j}^{1}-k_{i l}^{1} \frac{\partial \chi^{j}}{\partial y_{l}}\right)=0 \text {, in } Y \text {. }
$$

The solution $\chi^{j}$ can be determined up to an additive constant. Possible discontinuities of $k_{i j}^{1}$ are not considered here.

From Eq. (18), the necessary and sufficient condition for the existence of a $Z$-periodic solution of Eq. (16) is

$$
\begin{aligned}
\left\langle L_{y z} T_{3}\right. & \left.+\left(L_{x z}+L_{y y}\right) T_{2}\right\rangle_{Z} \\
& +\left\langle\left(L_{x y}+L_{y x}\right) T_{1}+L_{x x} T_{0}\right\rangle_{z}=f
\end{aligned}
$$

In view of $Y$-periodicity, on applying the operator $\langle\cdot\rangle_{Y}$ to both sides of Eq. (42) ones obtains 


$$
\left\langle\left\langle L_{x z} T_{2}+L_{x y} T_{1}+L_{x x} T_{0}\right\rangle_{Z}\right\rangle_{Y}=\langle f\rangle_{Y}=f
$$

Substituting the expressions (31), (39), and (40) for $T_{0}, T_{1}$ and $T_{2}$, respectively, into Eq. (43), and doing the algebra straightforwardly, leads to the homogenized equation, Eq. (3), whose components of the effective conductivity tensor are defined by

$\hat{k}_{i j}=\left\langle\left(k_{i j}-k_{i l} \frac{\partial \chi_{y}^{j}}{\partial z_{l}}\right)-\left(k_{i m}-k_{i l} \frac{\partial \chi_{y}^{m}}{\partial z_{l}}\right) \frac{\partial \chi^{j}}{\partial y_{m}}\right\rangle_{Y \times Z}$

Clearly, Eq. (44) can be rewritten as

$$
\hat{k}_{i j}=\left\langle k_{i j}^{1}-k_{i l}^{1} \frac{\partial \chi^{j}}{\partial y_{l}}\right\rangle_{Y},
$$

so that it incorporates the homogenization results from the previous level $Z$. It is worth noting that the effective tensor defined by Eq. (44) is symmetric and positive definite.

\section{APPLICATION TO A FUNCTIONALLY GRADED MATERIAL}

In this section the particular case of a periodic functionally graded material (FGM) is considered, whose coefficients $k_{i j}^{\varepsilon}(\mathbf{x})=k_{i j}(\mathbf{y}, \mathbf{z})$ are rapidly oscillating, isotropic $\left(k_{i j}^{\varepsilon}(\mathbf{x})=k^{\varepsilon}(\mathbf{x}) \delta_{i j}=k(\mathbf{y}, \mathbf{z}) \delta_{i j}\right)$, infinitely differentiable $\left(k_{i j}^{\varepsilon}(\mathbf{x}) \in C^{\infty}(\Omega)\right)$, and positive-definite $\quad\left(\exists c>0 \forall \boldsymbol{\eta} \in \square^{3}: k_{i j} \eta_{i} \eta_{j} \geq c|\boldsymbol{\eta}|^{2}\right.$, $\left.\boldsymbol{\eta}=\left(\eta_{1}, \eta_{2}, \eta_{3}\right),|\boldsymbol{\eta}|^{2}=\eta_{i} \eta_{i}\right)$. Because the coefficients are infinitely differentiable, it is not necessary to impose contact conditions on the constituents' interfaces.

The procedure of the previous section is here applied to a periodic FGM (Paulino et al., 2009) with $k^{\varepsilon}(\mathbf{x})=k(\mathbf{y}, \mathbf{z})$ being $Y \times Z \equiv[0,1] \times[0,1]$-periodic and given, with respect to some reference conductivity, by the trigonometric function

$k^{\varepsilon}(\mathbf{x})= \begin{cases}1+0.25 \sin \left(2 \pi \varepsilon^{-1} x_{1}\right) \sin \left(2 \pi \varepsilon^{-2} x_{1}\right), & x_{1} \in \Omega_{1} \\ 1, & x_{1} \in \Omega_{2}\end{cases}$

where $\Omega_{1}=[(n-1) \varepsilon,(n-0.5) \varepsilon], \Omega_{2}=[(n-0.5) \varepsilon, n \varepsilon]$, or

$k(\mathbf{y}, \mathbf{z})=\left\{\begin{array}{rr}1+0.25 \sin \left(2 \pi y_{1}\right) \sin \left(2 \pi z_{1}\right),\left(y_{1}, z_{1}\right) \in Y_{1} \times Z_{1}, \\ 1, & \left(y_{2}, z_{2}\right) \in Y_{2} \times Z_{2},\end{array}\right.$ where $Y_{1}=[n-1, n-0.5], Z_{1}=\left[(n-1) \varepsilon^{-1},(n-0.5) \varepsilon^{-1}\right]$, $Y_{2}=[n-0.5, n]$, and $Z_{2}=\left[(n-0.5) \varepsilon^{-1}, n \varepsilon^{-1}\right]$.

It is considered that the small parameter $\varepsilon$ takes values in the succession $\{1 / n: n \in \square\}$ such that $k^{\varepsilon}$ has periods $\varepsilon$ and $\varepsilon^{2}$. The local function is seen to depend on the fast variables $\mathbf{y} \equiv y_{1}$ and $\mathbf{z} \equiv z_{1}$ only. Figure 1 illustrates the rapidly oscillating character of the function $k^{\varepsilon}(\mathbf{x})$ for various values of $\varepsilon$.
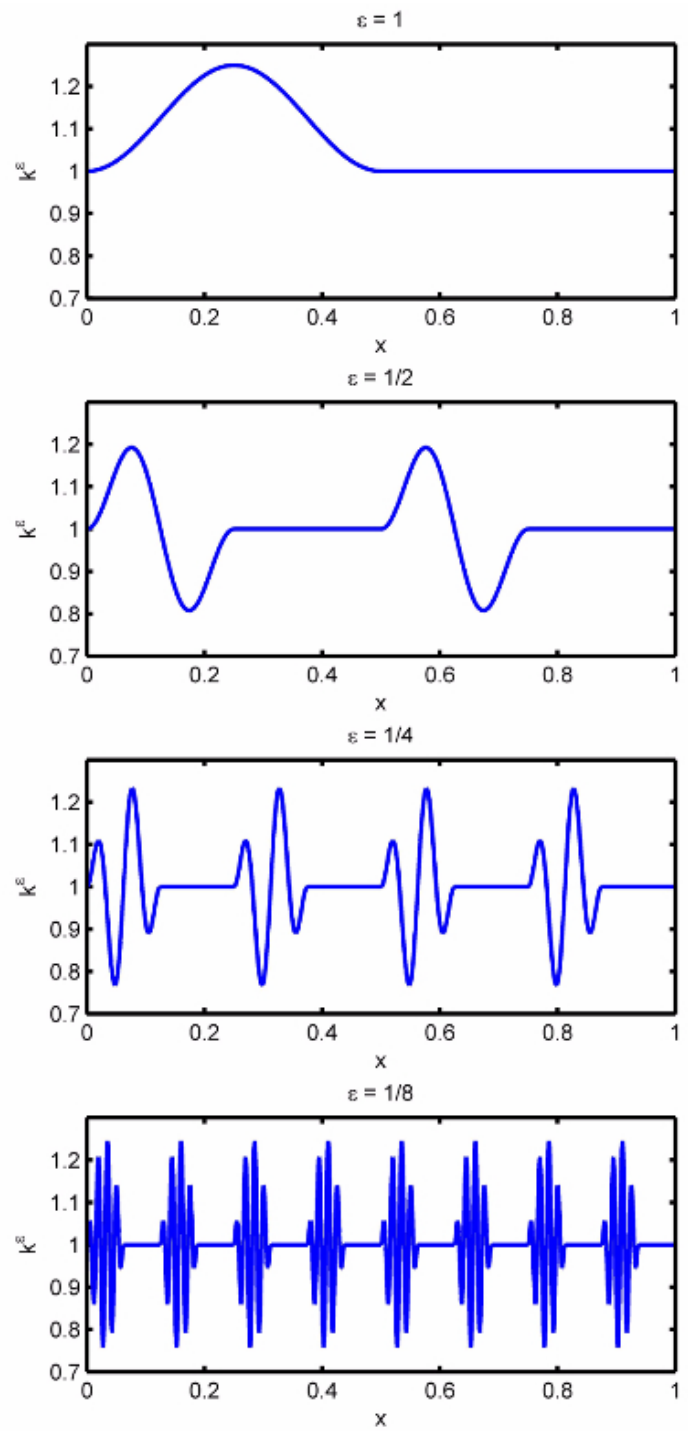

Figure 1. An illustration of the rapidly oscillating character of the function $k^{\varepsilon}(\mathbf{x})\left(\mathbf{x}=x_{1}\right)$ defined by Eq. (46).

Figure 2 illustrates the functionally graded behavior of the conductivity $k(\mathbf{y}, \mathbf{z})$ by considering the periodic part $[0,1] \times[0,1]$ of its domain of definition. For the FGM characterized by Eq. (47), a one-dimensional homogenization problem is, therefore, obtained, whose periodic cell solutions of 
Eqs. (22) and (41) must be found, in order to calculate the effective conductivity tensor given by Eq. (45).

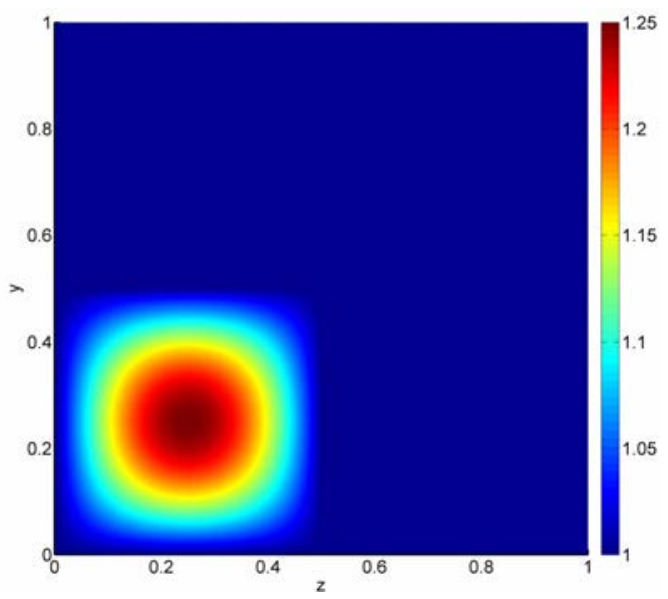

Figure 2. Periodic part $(n=1)$ of the domain of definition of the conductivity function $k(\mathbf{y}, \mathbf{z})$ of the FGM.

First Local Problem: For each $\mathbf{y} \in Y$, find the parametric family of $Z$-periodic functions $\left\{\chi_{y}^{j}\right\}_{y \in Y}$, such that

$$
\begin{gathered}
-\frac{d}{d z}\left(k_{1 j}-k_{11} \frac{\partial \chi_{y}^{j}}{\partial z}\right)=0, \quad \text { in } \mathrm{Z}, \\
\chi_{y}^{\ell}(0)=\chi_{y}^{\ell}(1), \\
\left\langle\chi_{y}^{\ell}\right\rangle_{Z}=0 .
\end{gathered}
$$

Integrating (48) in $z \in Z$, one obtains

$$
k_{1 j}-k_{11} \frac{\partial \chi_{y}^{j}}{\partial z}=C_{j}, C_{j} \text { const. } \forall j
$$

Thus

$$
\frac{d \chi_{y}^{j}}{d z}=k_{11}^{-1}\left(k_{1 j}-C_{j}\right)
$$

Integrating Eq. (52), from 0 to 1, yields

$$
\int_{0}^{1} \frac{d \chi_{y}^{j}}{d z} d z=\int_{0}^{1} k_{11}^{-1} k_{1 j} d z-C_{j} \int_{0}^{1} k_{11}^{-1} d z .
$$

Because $\chi_{y}^{\ell}(0)=\chi_{y}^{\ell}(1)$, Eq. (53) gives

$$
C_{j}=\left\langle k_{11}^{-1}\right\rangle_{Z}^{-1}\left\langle k_{11}^{-1} k_{1 j}\right\rangle_{Z}
$$

Substituting Eq. (54) into Eq. (52) yields

$$
\frac{d \chi_{y}^{j}}{d z}=k_{11}^{-1}\left(k_{1 j}-\left\langle k_{11}^{-1}\right\rangle_{Z}^{-1}\left\langle k_{11}^{-1} k_{1 j}\right\rangle_{Z}\right),
$$

so that Eq. (50) is satisfied.

With the solutions $\left\{\chi_{y}^{j}\right\}_{y \in Y}$ of the First Local Problem, it is possible to calculate the intermediate effective coefficients

$$
k_{i j}^{1}=\left\langle k_{i j}-k_{i 1} \frac{d \chi_{y}^{j}}{d z}\right\rangle_{Z}=\left\langle k_{i j}\right\rangle_{Z}-\left\langle k_{i 1} \frac{d \chi_{y}^{j}}{d z}\right\rangle_{Z},
$$

as a matter of fact, substituting Eq. (55) into Eq. (56) ones arrive at

$$
\begin{aligned}
k_{i j}^{1}=\left\langle k_{i j}\right\rangle_{Z} & -\left\langle k_{i 1} k_{11}^{-1} k_{1 j}\right\rangle_{Z} \\
& +\left\langle k_{i 1} k_{11}^{-1}\right\rangle_{Z}\left\langle k_{11}^{-1}\right\rangle_{Z}^{-1}\left\langle k_{11}^{-1} k_{1 j}\right\rangle_{Z} .
\end{aligned}
$$

One may verify that:

$$
\begin{aligned}
& \text { (i) } k_{i j}^{1}=k_{i j}^{1}(y) \in C^{\infty} ; \\
& \text { (ii) } k_{i j}^{1}=k_{j i}^{1} \text {; and } \\
& \text { (iii) } \exists c^{1}>0 \forall \boldsymbol{\eta} \in \square^{3}: k_{i j}^{1} \eta_{i} \eta_{j} \geq c^{1}|\boldsymbol{\eta}|^{2} .
\end{aligned}
$$

Second Local Problem: Find $\chi^{j}, Y$-periodic, such that

$$
\begin{gathered}
\frac{d}{d y_{i}}\left(k_{i j}^{1}-k_{i l}^{1} \frac{d \chi^{j}}{d y_{l}}\right)=0, \text { in } Y, \\
\chi^{j}(0)=\chi^{j}(1),
\end{gathered}
$$

The solution of this problem can be determined up to an additive constant.

The second local problem can be solved in a similar fashion, such that one can directly write

$$
\begin{aligned}
\hat{k}_{i j} & =\left\langle k_{i j}^{1}\right\rangle_{Y}-\left\langle k_{i 1}^{1}\left(k_{11}^{1}\right)^{-1} k_{1 j}^{1}\right\rangle_{Y} \\
& +\left\langle k_{i 1}^{1}\left(k_{11}^{1}\right)^{-1}\right\rangle_{Y}\left\langle\left(k_{11}^{1}\right)^{-1}\right\rangle_{Y}^{-1}\left\langle\left(k_{11}^{1}\right)^{-1} k_{1 j}\right\rangle_{Y} .
\end{aligned}
$$

In particular, for the case being considered, $k_{i j}(\mathbf{y}, \mathbf{z})=k(\mathbf{y}, \mathbf{z}) \delta_{i j}$, and Eq. (57) yields 


$$
k_{i j}^{1}=\langle k\rangle_{Z} \delta_{i j}-\left(\langle k\rangle_{Z}-\left\langle k^{-1}\right\rangle_{Z}^{-1}\right) \delta_{i 1} \delta_{j 1}
$$

As a consequence

$$
k_{11}^{1}=\left\langle k^{-1}\right\rangle_{Z}^{-1}, k_{22}^{1}=k_{33}^{1}=\langle k\rangle_{Z}, k_{i j}^{1}=0 \forall i \neq j
$$

Now with the help of math code, on substituting Eq. (47) for $k$ and Eq. (62) into the solution of the second local problem, Eq. (60), one finally determines the effective thermal conductivities,

$$
\begin{aligned}
\hat{k}_{11} & =\left\langle k_{11}^{1}\right\rangle_{Y}-\left\langle k_{11}^{1}\left(k_{11}^{1}\right)^{-1} k_{11}^{1}\right\rangle_{Y} \\
& +\left\langle k_{11}^{1}\left(k_{11}^{1}\right)^{-1}\right\rangle_{Y}\left\langle\left(k_{11}^{1}\right)^{-1}\right\rangle_{Y}^{-1}\left\langle\left(k_{11}^{1}\right)^{-1} k_{11}\right\rangle_{Y} \\
& =\left\langle\left(k_{11}^{1}\right)^{-1}\right\rangle_{Y}^{-1}=\left\langle\left\langle k^{-1}\right\rangle_{Z}\right\rangle_{Y}^{-1}=\left\langle k^{-1}\right\rangle_{Y \times Z}^{-1} \\
& =(0.228+0.75)^{-1} \\
& \approx 1.0225,
\end{aligned}
$$

$$
\begin{gathered}
\hat{k}_{22}=\hat{k}_{33}=\left\langle k_{22}^{1}\right\rangle_{Y}=\langle k\rangle_{Y \times Z} \\
=0.25+\frac{0.25}{\pi^{2}}+0.75 \approx 1.0253, \\
\hat{k}_{i j}=0, \forall i \neq j .
\end{gathered}
$$

\section{CONCLUSIONS}

In the present work the strong-form Fourier heat conduction equation for heterogeneous media with multiple spatial scales has been successfully derived using the method of reiterated homogenization. The effective coefficients can be calculated by solving two local or cell problems, while the macroscopic behavior is governed by the homogenized problem. To facilitate the physical understanding of the technique, an analytical solution is obtained for the heat conduction equation in a trigonometric functionally graded material. The approach presented here shall serve as a basis for future efforts to numerically compute effective properties of more complex materials with multiple spatial scales.

\section{ACKNOWLEDGEMENTS}

Support provided by $\mathrm{CNPq}$ - Brazilian National Council for Scientific and Technological Development (projects nos. 303208/2014-7 and 450892-2016-6) and CAPES - Brazilian Coordination for the Improvement of Higher Education Personnel (project no. 88881.030424/2013-01) is gratefully acknowledged. M. E. Cruz and J. B. Castillero are thankful to the Mechanical Engineering Program/COPPE/UFRJ for travel support.

\section{REFERENCES}

Allaire, G., and Briane, M., 1996, Multiscale Convergence and Reiterated Homogenisation, Proceedings of the Royal Society of Edinburgh: Section A Mathematics, Vol. 126, No. 2, pp. 297342.

Bakhvalov, N., and Panasenko, G., 1989, Homogenisation: Averaging Processes in Periodic Media, Kluwer Academic Publishers, Dordrecht, The Netherlands.

Bensoussan, A., Lions, J. -L., and Papanicolaou, G., 1978, Asymptotic Analysis for Periodic Structures, North-Holland Publishing Company, Amsterdam.

Mahamood, R. M., Akinlabi, E. T., Shukla, M., and Pityana, S., 2012, Functionally Graded Material: An Overview. Proceedings of the World Congress on Engineering - WCE 2012, Vol. III. London, U.K. Proceedings of the World Congress on Engineering 2012, July 4-6, London, U.K.

Panasenko, G. P., 2008, Homogenization for Periodic Media: from Microscale to Macroscale, Physics of Atomic Nuclei, Vol. 71, No. 4, pp. 681694.

Paulino, G. H., Silva, E. C. N., and Le, C. H., 2009, Optimal Design of Periodic Functionally Graded Composites with Prescribed Properties, Structural and Multidisciplinary Optimization, Vol. 38, No. 5, pp. 469-489.

Wargnier, H., Kromm, F. X., Danis, M., and Brechet, Y., 2014, Proposal for a Multi-Material Design Procedure, Materials and Design, Vol. 56, pp. 44-49.

Yin, H. M., Paulino, G. H., Buttlar, W. G., and Sun, L. Z., 2005, Effective Thermal Conductivity of Two-Phase Functionally Graded Particulate Composites, Journal of Applied Physics, Vol. 98, No. 6, pp. 063704. 